\title{
Identitätsbildung im Kunstunterricht
}

Selbstinszenierung, Rollenspiele und gestalterischer Ausdruck

Constanze Kirchner

Bildnerische Inszenierungen haben Hochkonjunktur: Handyfotos und Handyvideos dokumentieren auf Facebook oder anderen Onlineportalen das tägliche Bild vom Selbst - und tragen damit in hohem Maße zur Gestaltung der Identitätskonstruktion Jugendlicher bei. Dieses Phänomen wird im Kunstunterricht thematisiert, dessen Inhalt u.a. sowohl die inszenierten Alltagsbilder wie auch die künstlerischen Inszenierungen sind. Welche Merkmale eine Inszenierung bestimmen, welche gestalterischen Potenziale damit verknüpft und inwiefern den künstlerischen Inszenierungen Bildungspotenziale inhärent sind, sind Ausgangsüberlegungen, um das Verhältnis von Inszenierung und Identitätsbildung auszuloten. Im Kunstunterricht kann die intensive Reflexion über gestalterisches Inszenieren, performatives Handeln und Erleben ästhetisierter Inkohärenz usw. dazu beitragen, Handlungsoptionen zu eröffnen und die Verortung des Selbst zu unterstützen.

\section{Einleitung}

Was bedeuten der herrliche Blick vom Berggipfel nach einer anstrengenden Wanderung am Gipfelkreuz, die jubelnden Mengen des besuchten Konzerts oder der mit Freunden getrunkene Aperitif in der Abendsonne, wenn sie nicht mit anderen Personen geteilt werden können? Das Online-Publikum ist immer präsent und kann bei Bedarf an jedem Ereignis teilhaben. Die Inszenierung des Geschehens - zum Beispiel auf Facebook - führt zu einer Aufwertung des Alltags, denn die für das virtuelle Publikum hergestellte Szene macht das Erleben nochmals bedeutsamer. Der Jugendforscher Bernhard Heinzlmaier stellt fest, dass Online-

Inszenierungen und Selbstdarstellungen bei Jugendlichen außerordentlich beliebt sind: „Facebook ist eine ideale Bühne zur Selbstpräsentation“ (zit. nach Rella 2011). Die mediale Inszenierung führt zu einer Ästhetisierung und Steigerung des Erlebens, häufig gepaart mit verschiedenen Formen der Selbstinszenierungen. Heinzlmaier (2010, S. 142) spricht vom „homo aestheticus“, einem ästhetischen Wesen, das aus einer von Bildern geprägten Welt hervorgeht und dessen Vorreiter die Jugend ist. Mit den Augen zu denken, so Heinzlmaier, bedeutet, dass das unmittelbar erfahrene Sichtbare immer größere Relevanz erhält und die Form wichtiger als der Inhalt wird (vgl. ebd.).

Für Kinder und Jugendliche existieren viele neue Räume der Selbstinszenierung, die das Experimentieren mit sozialen Rollen sowie das Ausprobieren (alternativer) Lebenskonzepte ermöglichen. Die Lebenswirklichkeit Heranwachsender ist zunehmend mehr von solchen Selbstinszenierungen geprägt. Unterschiedliche kulturelle, soziale, weibliche oder männliche Lebensentwürfe werden im gesellschaftlichen Kontext erprobt, um sozialen Erwartungen zu entsprechen und um sich selbst positiv darzustellen, so Erving Goffman (1959/2003). Rollen werden nicht mehr sozial zugewiesen, sondern weitgehend frei gewählt. Das bedeutet, Person und Rolle müssen auf Grund von Selbstfestlegungen in Übereinstimmung gebracht werden. Grundlage dieser autonomen Identitätskonstruktion sind stabile, kontinuierliche Entwicklungslinien, die innere Einheitlichkeit und Kohärenz anstreben (vgl. Erikson 1973). Je größer die Spielräume für Identitätskonzeptionen sind, desto flexibler müssen Kinder und Jugendliche darauf reagieren. Dies heißt, die Suche nach einem kohärenten Selbstkonzept 
wird zunehmend komplizierter und muss aktiv - auch in schulischem Kontext - unterstützt werden. So genannte „Patchworkidentitäten“(Keupp u.a. 1999; Keupp 2009) bedürfen der Reflexion und Positionierung, der kritischen Prüfung für das eigene Selbst. Die Frage „Wer bin ich?" ist eine in der Adoleszenz entscheidende und kann beispielsweise durch „Selbsterzählungen“ und „Selbstbindung“ (vgl. Nunner-Winkler 2009) gefördert werden. Zugleich gilt es Aktionsfelder oder Bühnen zu eröffnen, die Erprobungsräume auch für divergierende Lebenskonzepte schaffen. Mit Hilfe von versuchsweisen Selbstinszenierungen und Rollenspielen lassen sich Handlungsoptionen ausprobieren und ggf. verfügbar machen. Performatives Handeln erlaubt zudem, inkohärente Selbstkonzepte - Patchwork-Identitäten $\mathrm{zu}$ inszenieren und damit auch zu reflektieren.

\section{Identitätsbildung als Inszenierungsleistung}

Wird ein Ereignis bildnerisch konkretisiert und beispielsweise dem Facebook Publikum vorgestellt, erhält es performativen Charakter. Es wird mit gesteigerter Intensität und Aufmerksamkeit wahrgenommen. „Ob etwas als Inszenierung zählt, hängt immer mit davon ab, wann es und für wen es als Inszenierung zählt", so Martin Seel (2001/ 2013, S. 58), der das Inszenieren als besonderes „Erscheinenlassen“ definiert und davon ausgeht, dass zwar nicht ,alles im Leben (...) Inszenierung“ ist, aber ,,alles in der Kunst (...) - wie im Leben Gegenstand von Inszenierung werden“"kann (Seel 2001/ 2013, S. 61). Seel beschreibt eine Inszenierung als ,ein artifizielles Geschehen, das sich als auffälliges Ereignis vom nicht inszenierten Geschehen abgrenzen lässt" (ebd., S. 52 f.), das intentional erzeugt wird und eines Publikums bedarf (ebd., S. 49 ff.). „Inszenierungen sind absichtsvoll ausgeführte oder eingeleitete sinnliche Prozesse“ (ebd., S. 49). Gleich ob künstlerische oder alltägliche Inszenierungen - ihre Sinnhaftigkeit liegt im Herstellen einer besonderen Gegenwart, die als symbolische Vergegenwärtigung (ebd., S. 55) sinnliche Prägnanz, verknüpft mit ästhetischer Wahrnehmung und Erfahrung, aufweist (ebd., S. 56). Inszenierungen sind aus dieser Perspektive grundsätzlich ästhetisch fundiert, sie erwirken Intensität und Aufmerksamkeit, um etwas herauszustellen und um es spürbar zu machen (ebd., S. 57). Im Gegensatz zur Alltagsinszenierung definiert Seel die künstlerische Inszenierung als Präsentation mit gesellschaftlich bedeutsamem Sinn - künstlerische Inszenierungen „,bieten Gegenwarten dar. Sie sind Imaginationen menschlicher Gegenwarten - ob dies nun vertraute oder unvertraute, vergangene oder zukünftige, wahrscheinliche oder unwahrscheinliche Weltverhältnisse sind“ (ebd., S. 58).

In ihrem Buch „Ästhetik des Performativen“ schließt Erika Fischer-Lichte (2004) an die Ausführungen Seels an und betont ebenfalls den Kampf um die Aufmerksamkeit als ein Merkmal von künstlerischer Inszenierung (vgl. ebd., S. 318 ff.). Konkreter als in der Definition von Seel fasst sie die Inszenierung nicht nur als Darstellungsweise, die etwas besonders auffällig in Erscheinung treten lässt, sondern als Erzeugungsstrategie (vgl. ebd., S. 324), das heißt, die Inszenierung ist eine aktive, intentionale Hervorbringung eines Ereignisses für ein Publikum. Erst die „Wahrnehmung der Zuschauer und ihre Reaktion auf das Wahrgenommene“ konstituieren die Inszenierung, so Fischer-Lichte (ebd., S. 327), „es ist die Inszenierung, welche eine Situation entwirft, in der sich etwas ereignen kann“ (ebd., S. 328). Ausgehend von dem Zusammenspiel von Akteuren und Publikum in der Bildenden Kunst sieht Fischer-Lichte Grenzüberschreitungen und Selbstinszenierungen der Künstlerinnen und Künstler als paradigmatisch für eine Bestimmung jüngster künstlerischer 
Ausdrucksformen seit den 60er Jahren des 20. Jahrhunderts. Fischer-Lichte charakterisiert solche Werke als ästhetische Ereignisse, die unmittelbar aus ästhetischen

Inszenierungsprozessen resultieren und ästhetische Erfahrung u.a. durch permanente Grenzüberschreitungen auslösen (vgl. ebd., S. 318 ff.). Mit der Grenzüberschreitung verbunden ist die kontinuierliche Neudefinition des Selbst, und zwar in Prozessen besonderer „Intensität und Auffälligkeit“ (ebd., S. 359). Anthropologisch gewendet bedeutet dies nach Helmuth Plessner, so Fischer-Lichte, dass der Mensch „nur er selbst werden (kann), wenn er sich permanent neu hervorbringt, sich ständig verwandelt, immer wieder Schwellen überschreitet (...) Es ist unser Leben, das in der Aufführung in Erscheinung tritt, gegenwärtig wird und vergeht" (ebd., S. 359).

Diese anthropologische Facette im Begriff der Inszenierung als (Lebens-) gestalterischem Mittel spiegelt sich nicht nur in Inszenierungen auf verschiedenen Internetportalen und in anderen Medien, sondern auch im alltäglichen Rollenverhalten, im Erproben von Lebensstilen oder Moden. Die spielerische Selbstdarstellung will weder täuschen noch lügen, sondern lediglich gestalten (vgl. hierzu Früchtl/ Zimmermann 2013, S. 16, mit Bezug auf Schulze 1999). Früchtl/ Zimmermann (2001/ 2013) beleuchten die „Ästhetik der Inszenierung“ als ein gesellschaftliches, individuelles und kulturelles Phänomen, das einen Wandel der Identitätsbildung skizziert: „Die Bildung von Ich-Identität gestaltet sich (...) nicht mehr als anstrengender Prozess der Selbst-Suche, sondern kehrt sich dem Erscheinungsbild zu, der Sphäre der äußeren Gestaltung, deren Regentin wiederum die Mode ist" (Früchtl/ Zimmermann 2013, S. 25). Das Anders-Sein, das Sich-Herausheben aus der Gemeinschaft, das Erregen von Aufmerksamkeit und Erzielen dramatischer Effekte bedarf des Publikums und damit der (medialen) Inszenierung (ebd., S. 26 ff.).

Wolfgang Welsch (1993, S. 76) betont in seiner Schrift „Ästhetisches Denken“, dass der Umgang mit aktueller Kunst Orientierungshilfe in der Gegenwart sein kann und dadurch Handlungskompetenz auszubilden vermag (vgl. ebd., S. 76). Dies gilt freilich nicht nur für die Bildende Kunst sondern ebenso für die Literatur, die Musik oder andere kulturell gestaltete Phänomene, die Lebensformen spiegeln und somit Vorbildfunktion für die individuelle Existenz haben können. Mit anderen Worten: Die ästhetische Erfahrung soll helfen, sich in der Lebenswelt zurechtzufinden und unterschiedliche Wirklichkeitsmodelle und Identitätskonstrukte auszubilden. Welsch sieht den Menschen als ,hartnäckigen Identitätskonstrukteur', der Überschneidungen, Bezugnahmen und Verbindungen zwischen seinen Teilidentitäten herstellen muss, wobei ihm die Beschäftigung mit anderen, fremden und neuen Lebensentwürfen hilft: „Identität ist immer weniger monolithisch, sondern nur noch plural möglich. Leben unter heutigen Bedingungen ist Leben im Plural, will sagen: Leben im Übergang zwischen unterschiedlichen Lebensformen" (Welsch 1993, S. 171). Welsch meint, die gesellschaftliche Entwicklung zur Pluralität sei unverkennbar und es komme darauf an, „,Formen zu finden und auszubilden, in denen diese Pluralität vollziehbar und in Übergängen mit neuen Identitätsfindungen lebbar wird. Die Kunst spiegelt solche Identitätsvervielfachung seit langem mit besonderer Eindringlichkeit wider" (ebd., S. 171 f.).

Das Ziel der Identitätsentwicklung ist nach Welsch, ,seine Identität so auszubilden, dass sie der aktuellen Pluralität gewachsen, Identität in Übergängen ist" (ebd., S. 197). Dem halten Keupp u. a. (1999, S. 59) entgegen, dass das Kohärenzprinzip für die Identitätsbildung nicht zur Disposition gestellt werden dürfe, und dass es Ziel sein müsse, Ankerplätze für die Identitätsbildung zu schaffen, die eine Identitätskonstruktion als Sinnkonstruktion unterstützen. Kunst, Musik, Literatur und Religion können solche Ankerplätze bieten. 


\section{Identität als Kohärenz mit sich selbst}

Der Blick auf die unterschiedlichen Forschungsansätze der letzten zwei Jahrzehnte zur Identität zeigt eine facettenreiche und inhomogene Diskussion mit zahlreichen Modellvorstellungen zur Identitätsentwicklung (vgl. u.a. Hafeneger 2003). Es ist die Rede von der Identität, dem Ich, dem Selbst, dem Subjekt, dem Selbstbild, dem Selbstkonzept, von Identitätsstrukturen, Identitätszuständen, Identitätsphasen usw. Markus P. Neuenschwander (1996) spricht von einem angeborenen, eigenschaftslosen, nicht unmittelbar erfahrbaren Kern (Subjekt), der persönliche, soziale, materielle, abstrakte und handlungsbezogene Identitäten mit bestimmten Einstellungen, Werten, Eigenschaften ausbildet (vgl. ebd., S. 14 f.). Einzelne Identitätsschemata werden für die eigene Identität bewertet und in das Selbstbild eingeordnet bzw. integriert. Das Selbstbild bzw. Selbstkonzept entstehe durch die Entwicklung und Reflexion dieser Identitätsschemata. Eindrücke, Erfahrungen und Erlebnisse werden gesammelt, im Hinblick auf die eigene Identität bewertet und in das Selbstkonzept eingegliedert. Das heißt, Subjekt, Identität und Selbstbild/ Selbstkonzept bilden eine Inklusionshierarchie, so Neuenschwander (vgl. ebd. S. 14 ff.).

Für den Kunstunterricht ist in diesem Zusammenhang interessant, dass die Identitätsentwicklung von einem dynamischen Einpassen sinnlicher Erfahrungen und ästhetischer Wahrnehmungen in das Selbstbild begleitet wird und dass diese zugleich in einen permanenten Selbstreflexionsprozess eingebunden sind. Als wesentliche Kriterien der Identitätsbildung sieht Karl Haußer (1995) insofern auch die Fähigkeit zur Selbstwahrnehmung, Selbsteinschätzung und Selbstbewertung - Kompetenzen die gerade mit der ästhetischen Bildung entwickelt werden. Darüber hinaus liegt nach Haußer in der subjektiven Bedeutsamkeit, die konstitutiver Bestandteil ästhetischer Erfahrung ist, die Chance zur Identitätsentwicklung, weil nur durch Betroffenheit Reflektieren und In-FrageStellen angeregt werde: „Was einen emotional nicht berührt ..., wird auch nicht identitätsrelevant“" (Haußer 1995, S. 9).

Versteht man Identität als innere, selbst konstruierte, dynamische Organisation von Trieben, Fähigkeiten, Überzeugungen und individueller Geschichte (Marcia 1980, zit. nach Haußer 1995, S. 3), die sich im steten Austausch mit dem Innen und dem Außen entwickelt und entsprechende Relationen zwischen den intrapersonellen Teilidentitäten und dem sozialen Umfeld aufbaut, existiert kein fester Identitätskern. Stattdessen ist Identität nur im hermeneutischen Selbstauslegungsprozess erfahrbar, wobei sowohl Individualität und Konsistenz innerhalb der Innenperspektive als auch Stabilität und Wandel (Kontinuität) angestrebt werden (nach Frey/ Haußer 1987, S. 17). Benno Hafeneger (2003) betont besonders die intersubjektive Konfiguration des Selbst als oszillierende Wechselwirkung in Interaktion mit der Umwelt durch wechselseitige Anerkennung (Hafeneger 2003, S. 43 ff.). Diverse plurale persönliche und soziale Identitätsinhalte bestimmen die Teilidentitäten, die wiederum das Selbstbild/ Selbstkonzept konstituieren. Interaktion mit der Umwelt (Identifikation, Anpassung und Reflexion) bedeutet immer eine Restrukturierung des Selbstkonzeptes. Gravierende Um- und Neustrukturierungen des Selbstbildes, wie dies im Jugendalter entwicklungsbedingt meist geschieht, können Krisen auslösen. Gelingt keine Integration des Neuen in das vorhandene Selbstkonzept, droht Identitätsdiffusion als intrapersonaler Konflikt, oder aber die Krise wird bewältigt, und die Identitätsentwicklung schreitet voran. 
Die lebensweltliche Disparität verschärft einerseits die Frage nach der inneren Konstruktion von Kohärenz, weil die Verarbeitung von Erfahrungszerrissenheit mit Blick auf die Integrität und Unversehrtheit des Subjekts in den Blick rückt, zugleich bietet sich andererseits die Chance der Kontingenz - Optionen für mögliche, selbst konstruierte und selbst bestimmte Lebensentwürfe eröffnen sich. Erst die Vielfalt des Selbsterlebens macht Kohärenz und Identität möglich (Keupp u. a. 1999, S. 69). Die zentrale Identitätsfrage lautet dann „Wer bin ich im Verhältnis zum Anderen, zum Fremden, zum Neuen etc.?" So ist das Identitätskonstrukt untrennbar mit dem Konzept der Alterität verwoben (vgl. ebd.). Gemeint ist damit das Erkennen des Selbst im Anderen, im Fremden, im Gegenüber (Person,

Kunstwerk, Text, Musikstück, Symbol usw.) einerseits als ein Wiedererkennen sowie anderseits als die Bereitschaft, im Anderen, Fremden sich selbst neu zu begreifen, wobei immer ein Rest des Nicht-Verstehens bleibt (vgl. Jauß 1994, S. 23).

\section{Rollen- und Selbstinszenierungen in der Kunst}

Stereotypen zu erkennen - Charakteristika von Menschen in Haltung und Ausdruck, nach ethnischen Differenzen oder in Berufsfeldern -, Theater zu spielen und kurzfristig eine andere Identität anzunehmen, ist eine lustvolle Beschäftigung für Kinder, Jugendliche und Erwachsene. So lassen sich auch in der Bildenden Kunst zahlreiche Beispiele finden, die Selbstinszenierungen, Rollenbilder, Verkleidungen, Maskeraden, veränderte Identitäten usw. zeigen, denken wir nur an Dürers Selbstbildnis, die Selbstporträts von Rembrandt, die Tableaux vivants und historisierende Fotografien des 19. Jahrhunderts. Speziell die Fotografie eröffnet vielfältige Möglichkeiten des Festhaltens temporär angenommener Rollen, Selbstinszenierungen, Bildparaphrasen oder nachahmender Adaptionen bestimmter Bilder. Das Spiel mit den Identitäten findet nicht nur wegen der fotografischen Möglichkeiten besonders in der Kunst des 20. Jahrhunderts seinen Raum, sondern auch weil sich das Befragen von biologischen, sozialen, ethnischen, religiösen und kulturellen Lebensentwürfen im heterogenen Feld des 20. und 21. Jahrhunderts zunehmend steigert.

Mit der fortschreitenden Frauenemanzipation gilt in der Kunst des 20. Jahrhunderts der Geschlechtsidentität und den feministisch geprägten Weiblichkeitskonzepten besondere Aufmerksamkeit. Während zunächst vorrangig geschlechtsspezifische Rollenzuweisungen wie maskuline und/ oder feminine Rollen künstlerisch thematisiert wurden, stehen heute Genderfragen und geschlechtsunabhängige Identitätskonzepte im Vordergrund künstlerischer Intentionen. 1921 fotografiert Man Ray seinen Künstlerkollegen Marcel Duchamp, der sich als Frau verkleidet hatte: Es ist ein Porträt von Rrose Sélavy - Duchamps Pseudonym. Eva und Adele, ein Künstlerduo, das seit den 90er Jahren im öffentlichen Raum glatzköpfig, stark geschminkt und affektiert-stilisiert gekleidet auftritt, verbirgt seine wahre Geschlechtsidentität: Zwar ist ihr Äußeres weiblich definiert, doch sie unterstützen eine frei wählbare Geschlechtszuordnung. Mit „Over the Boundaries of Gender“, so einer ihrer Titel, kennzeichnen sie sich als lebendes Artefakt, das keinen Rückschluss auf ihr biologisches Geschlecht erlauben soll (siehe hierzu: Drühl 2012).

Die Inszenierung männlicher und weiblicher Identitäten und der Wechsel des Geschlechts in einer anderen Rolle finden sich in zahlreichen Beispielen Bildender Kunst, insbesondere in den 1970er Jahren und Anfang der 1980er Jahre, als der feministische Kampf um die Gleichberechtigung von Mann und Frau einen weiteren Höhepunkt erreichte. 
Jürgen Klauke thematisiert die „Durchlässigkeit geschlechtsspezifischen Auftretens“ mit seinen Self Perfomances, die er mit eindeutig femininen wie maskulinen Attributen bestreitet. Seine künstlerischen Aktionen intendieren die Reflexion sexueller Identität und werfen die Frage nach dem sozial determinierten Geschlecht auf. Er wendet sich gegen tradierte Geschlechtsrollenzuweisungen und gegen geschlechtskonforme Lebensmuster (Holschbach 2011, S. 206). Die Befragung der eigenen Person als Mensch, als Mann oder Frau, als Künstler usw. steht im Mittelpunkt von zahlreichen Künstler-Selbstporträts, die sich in ihren Identitätskonstruktionen reflektieren.

Urs Lüthi beispielsweise experimentiert mit Selbstdarstellungen, die immer wieder einer Selbstbefragung und Selbstpositionierung gleich kommen. Er stellt sich mit fließenden femininen und maskulinen Gesichtszügen dar und versucht Emotionen deutlich werden zu lassen. Auch Andy Warhol, dessen narzisstische Selbstinszenierung weitreichend biografisch belegt ist, stellt mit seinen Polaroid-Fotos die Frage „Wer bin ich?“ Da die Anteile des eigenen Selbst erst im Spiegel des Anderen bewusst werden, zeigt Warhol eine weibliche Seite im männlichen Körper. Die fotografische Selbstdarstellung als weibliche Person in Männerkleidung hat Warhol zusammen mit dem Fotografen Christopher Makos perfektioniert und eine Serie von „Cross-Dressing-Performances“ entwickelt (Holschbach 2011, S. 194).

Identitätskonstruktionen basieren auf verschiedenen Bereichen, die identitätsstiftend im Leben wirken: Familiäre Kontexte, Geschlechterrollen, ethnische Identität, Heimat, private und öffentliche Rollen, Berufsbilder, soziale Milieus, Peergroups und andere Gruppenzugehörigkeiten sind ebenso ausschlaggebend für individuelle Lebensentwürfe wie biologische und biografische Gegebenheiten, Wissen und Können, Erfahrungen,

Beziehungen, Vorstellungen usw. Die verschiedenen Darstellungsformen sind Repräsentanten des veränderbaren Selbstbildes - nicht Ausdruck der Persönlichkeit. So zeigen beispielsweise die Arbeiten von Tracy Emin und Cindy Sherman zwei völlig unterschiedliche weibliche Lebensmuster - Freiheit und sexuelle Unabhängigkeit, gepaart mit seelischem Chaos, in vielen Installationen von Emin - sowie etwa die vom Einkauf wiederkehrende, entnervte Hausfrau am Herd im Film-Still von Sherman. Cindy Shermans Untitled Film Stills lassen die Erinnerung an bekannte Filme aufscheinen, doch konkrete Vorbilder für ihre Szenen gibt es nicht. Sie schlüpft in verschiedene Rollen, erprobt männliche und weibliche Rollenmuster mit bildnerischen Mitteln wie Kleidung, Körper, Mimik und Gesten, so dass - überzogen dargestellte - Stereotypen prägnant dargestellt werden. Sie schafft Protagonisten für eine narrative Erzählung, die beim Betrachter dadurch entsteht, dass sich eine Vorstellung von einer Szene, einer Lebenssituation, einer bestimmten Zeit etc. entwickelt.

Markant und treffend fotografiert August Sander zu Beginn des 20. Jh.s Personen in ihren Berufen. Während Sander typisierend die charakteristische Gruppenzugehörigkeit betont, ist die niederländische Fotografin Rineke Dijkstra dafür bekannt, dass sie die Individualität der einzelnen Personen herausarbeitet und deren Verletzlichkeit in sachlicher Distanz hervorscheinen lässt (vgl. K+U 2009). Kulturelle, ethnische oder soziale Lebenswelten stellt Jeff Wall mit seinen Fotografien in das Zentrum seiner künstlerischen Intentionen. Als Großbilddia im Leuchtkasten präsentiert, inszeniert er häufig Szenen im Niemandsland, an der Autobahn oder inmitten beliebiger Ödnis am Stadtrand. Die scheinbare Zufälligkeit der Aufnahmen täuscht jedoch: Jeff Wall wählt die Orte seiner Fotoinszenierungen mit Bedacht aus und arbeitet mit Schauspielerinnen, die in Szene gesetzt werden. Jeff Wall entwirft mit seinen Fotos Lebensgeschichten - und gibt Anstöße, diese zu reflektieren. Was empfindet oder denkt die Person, was haben die dargestellten Menschen miteinander zu tun? Wie leben

Kirchner, Constanze: Identitätsbildung im Kunstunterricht. Selbstinszenierung, Rollenspiele und gestalterischer Ausdruck. In: Hagedorn, Jörg (Hg.): Jugend, Schule und Identität. Selbstwerdung und Identitätskonstruktion im Kontext Schule. Springer VS: Wiesbaden 2014, S. 503-517 
sie in einem brachliegenden, identitätslosen Landstrich, in dem die Natur gerade die Bauschuttreste und den Abfall überwuchert? Tristesse betont die schwierige Lebenssituation jener Menschen, die entwurzelt - in Folge moderner Zivilisation und Industrialisierung - in irgendeiner halbwegs urbanen Gegend leben.

\section{5. Ästhetische Bildung im Kunstunterricht}

Die Offenheit heterogener und pluraler kultureller bzw. gesellschaftlicher Entwürfe verlangt von Kindern und Jugendlichen eine ungleich größere Anstrengung als bisher, um sich in den disparaten Lebenswelten zurecht zu finden und die so genannten Teilidentitäten in Einklang miteinander zu bringen. Zugleich bieten sich jedoch auch andererseits offene Optionen für mögliche, selbst konstruierte und selbst bestimmte Lebensentwürfe. Das heißt, einerseits wird es möglich, tradierten Rollenerwartungen zu entfliehen, andererseits müssen jedoch neue Normen und Werte selbst erarbeitet werden. Hierbei kann der Kunstunterricht die Schülerinnen und Schüler unterstützen, indem einerseits vielerlei Lebensmuster zur Reflexion angeboten werden, andererseits der eigene Ausdruck zur Selbstreflexion im praktischen Tun animiert.

Inszenierungen sind notwendiger Bestandteil der Identitätsentwicklung und beinhalten aktives gestalterisches Handeln, das mit ästhetischer Erfahrung und ästhetischem Erleben verwoben ist. Schule kann einen performativen Handlungsraum für Selbstkonstruktions- und Identitätsprozesse bieten, die narrativ, performativ, rituell, gestalterisch hergestellt, inszeniert und erprobt werden können. Speziell im Kunstunterricht kann die Bildung von Ich-Identität unterstützt werden, denn jeder bildnerisch auffällig zur Erscheinung gebrachte Gegenstand, jedes wie auch immer inszenierte Ereignis, ist eine symbolische Vergegenwärtigung von Erlebtem oder Imaginiertem. Entscheidend ist nicht die Frage, ob die Herstellung von Sinn, Bedeutung und Kohärenz durch gestalterische Inszenierungen im Vordergrund der Identitätsbildung steht oder ob vielmehr erst das performative Tun sowie das (krisenfreie) Erfahren und Erleben einer ästhetisierten Inkohärenz bzw. Nicht-Identität die Identitätsbildung geradezu herausfordert, sondern entscheidend ist die intensive Reflexion über diese Prozesse.

Über Inszenierungen und Gestaltungsvarianten zu reflektieren, ist ein Nachdenken mit dem Ziel eines Erkenntnisgewinns, der alternative Handlungsmöglichkeiten eröffnet. Es geht jedoch nicht darum, eindeutige Selbstbilder zu erarbeiten, sondern vielmehr um das Entwickeln der Fähigkeit, Handlungsoptionen anderer Personen antizipieren zu können. Wenn es uns also im Kunstunterricht gelingt, einen von Spiel und bildnerischem Ausdruck getragenen Raum zu eröffnen, der das aktive Sich-selbst-verorten erlaubt, können wir in hohem Maße helfen, die Integrität des Subjekts und damit die Identitätsentwicklung zu stärken. Im Kunstunterricht können bildnerische Umsetzungen vom Selbstkonzept und vom Anderssein Reflexionsangebote darstellen, um intersubjektiv verbindliche Lebensentwürfe zu thematisieren. Dabei können die Reflexionsgegenstände sowohl künstlerische Inszenierungen als auch inszenierte Alltagsereignisse sein, die im besonderen Schülerinteresse liegen.

Hinzu kommt ein weiterer identitätsbildender Aspekt, der speziell mit ästhetischen Bildungsprozessen einhergeht, nämlich das Entwickeln von Ich-Stärke durch das Hervorbringen von Gestaltetem. Wirklichkeit wird in besonderem Ausmaß aktiv auf ästhetische Weise geformt: Das Inszenieren, Musizieren, Tanzen, Gedichte verfassen, 
Zeichnen, Malen, Bauen oder Konstruieren erfordert das Aktivieren innerer Vorstellungen, innerer Klänge, innerer Dialoge, innerer Bilder, Fantasien und Träume, die geordnet und in eine gestaltete Form gebracht werden. Es wird etwas geschaffen, wodurch

Selbstwirksamkeitsgefühle entstehen.

Die Inszenierung des Selbst benötigt Rückmeldungen durch das soziale Umfeld, also Zuschauer. Das ist die Internetcommunity, das sind die Peers, Sportgruppen, Vereine, Musikszenen, Klassenkameradinnen und -kameraden, aber auch Lehrerinnen und Lehrer, Eltern, Nachbarn etc. Wer seine Rolle gut spielt, erhält Zuspruch und Anerkennung, Spielverderber werden aus dem sozialen Netz ausgeschlossen oder suchen sich Nebenschauplätze, die Aufmerksamkeit versprechen. Der Kunstunterricht bietet einen geschützten Rahmen, um im Kontext bestimmter Aufgabenstellungen die eigenen Lebensentwürfe und Rollenerwartungen bewusst zu machen und zu reflektieren. Diese Inszenierungen ermöglichen es zugleich, in neue Rollen zu schlüpfen und diese auszuprobieren, gestalterisch zu pointieren oder zu karikieren.

Der Kunstunterricht kann zwar keine sozialen Defizite oder Diffusionen im Identitätsprozess ausgleichen, doch gewiss ist, dass materiale und handwerkliche Anforderungen für die Identitätsentwicklung der Schülerinnen und Schüler bedeutsam sind. Denn die Erfahrung von Selbstwirksamkeit durch die gestalterische Bearbeitung eines Materials stärkt das Ich, darüber hinaus ermöglicht der gestalterische Ausdruck Selbstreflexion, weil die Darstellung verdichtet und in simultaner Präsenz als materiales Gegenüber dasteht und mit Distanz betrachtet werden kann. Die Selbstinszenierung und das Rollenspiel bergen pädagogische Chancen, das eigene Ich im Anderen zu erkennen. Angebote, sich im Anderen zu sehen oder das Selbst im Neuen, Fremden zu spiegeln, kann der Kunstunterricht bieten, indem einerseits die Auseinandersetzung mit Alltagsbildern, Werbung, Kunstwerken usw. gefördert wird, andererseits aber auch die Frage nach dem Ich - woher komme ich, wohin gehe ich, wer bin ich? - in Gestaltungsprozessen reflektiert wird. Das Moment der gestalterischen Produktion kann die aktive Aneignung anderer Lebensentwürfe beinhalten und schärft dadurch das Bewusstsein für verschiedene kulturelle, soziale oder geschlechtsspezifisch geprägte Lebensmuster, wenn diese als gestalterischer Ausdruck reflektiert werden. Kinder und Jugendliche können jemand anderen, aber auch sich selbst in anderen Rollen spielen - gleich ob in realen oder virtuellen Welten. Zweifellos können sie dabei unterscheiden, ob und inwiefern sie eine Rolle spielen, ob Sie etwas von sich preisgeben oder etwas darstellen, was ihnen völlig fremd ist. Wenn man sie darum bittet, können Jungen das Verhalten von Mädchen imitieren und umgekehrt, Jungen können wie Mädchen zeichnen, Mädchen wie Jungen - dies zeigen vielfältige Versuche im Kunstunterricht. Letztlich muss die Person selbst entscheiden, welchen Faktoren eine Identitätsrelevanz zukommt und welche begründeten Merkmale für das eigene Selbstverständnis bestimmend sind (Nunner-Winkler 2009, S. 15).

Während im Unterricht mit Grundschulkindern das Selbstporträt, verschiedene Varianten des szenischen Spiels wie Schatten- oder Maskenspiel, das Kennenlernen von anderen Personen, das Verkleiden oder das Anlegen biografischer Sammlungen im Vordergrund des gestalterischen Selbstausdrucks und Rollenspiels stehen, können die Rollenübernahme und die Selbstinszenierung mit Jugendlichen das Fremde, das Surreale und Mögliche, den Symbolwert von Ausdrucksfarbe, Proportion und Komposition, das collageartige Zusammenfügen, das Ironisieren, Karikieren und Übertreiben verstärkt als gestalterische Ausdrucksmittel einsetzen. Speziell in der Adoleszenz, einer Phase, in der Jugendliche sich stark mit der eigenen Identität beschäftigen, in der sie unterschiedliche Daseinsformen, 
Rollen, Lebensstile erproben und in der sie auf der Suche nach dem Selbst sind bzw. in der sie sich im gesellschaftlichen Kontext selbst verorten müssen, bietet es sich an, sie mit ästhetischen Mitteln auf dem Weg zur Identitätskonstruktion zu unterstützen. Wer ich bin, zeigt sich auch in Mode, Kleidung, Frisur ebenso wie in der Ausgestaltung des Zimmers, der virtuellen Umgebung oder der Wahl der Freunde, der Musikszenen, Vereine usw. Herauszufinden, wer ich (die Schülerin/ der Schüler) vielleicht auch noch sein könnte und wer der Andere ist, kann Ziel solchen Kunstunterrichts sein, der das gestalterische Inszenieren einer Person thematisiert.

\section{Bildwissen als Grundlage der Identitätsentwicklung}

Kunstwerke bieten nicht nur Lebensentwürfe an, sie treffen zudem das Selbstverständnis des Rezipienten: „Was dabei in den Blick gerät, ist ein Problem der Grenze des Selbstgefühls und des Ästhetischen“" (Schuhmacher-Chilla 1995, S. 71). Bildliches Wissen spiegelt sich in sinnlichen Erfahrungen, szenischen Episoden wider, die wir als Erinnerung in uns tragen. Es sind in das Gedächtnis eingeprägte Episoden unserer Lebensgeschichte: „Diese Bilder bestimmen unser Selbst, und sie lassen uns in uns heimisch werden. Wenn wir uns fragen, welches unsere erste Erinnerung ist, dann tritt in unsere Vorstellung ein Bild, und dieses Bild bezieht sich auf einen bestimmten Ort und ein bedeutsames Ereignis, das uns nicht mehr loslässt. Damit sich unser Selbstwissen, unsere Identität, ausprägen kann, müssen wir uns bis an die Grenzen möglicher Erfahrungen vorwagen. ... Erst an den Grenzen erkennen wir uns, und diese Grenzerfahrungen in Lust und Schmerz, Liebe und Verzweiflung sind es, die immer als Bilder in unseren Erinnerungen bleiben. Bildliches Wissen als Erinnerungswissen ist also grundlegend für das Selbstwissen“ (Pöppel 2000, S. 26). Pöppel hebt die zentrale Bedeutung bildhaften Wissens für die Identitätsbildung hervor: Gerade das implizite Handlungswissen und das Bildwissen seien ich-nahe Wissensformen, die nachhaltig die Persönlichkeitsentwicklung bestimmen. Das Erinnerungswissen (episodische Erinnerung) weist nach Pöppel (2000) die größte Ich-Nähe und Unmittelbarkeit auf. „Das Wissen um unsere Identität gründet auf Bildern unserer Lebensgeschichte, die in höchstem Maße subjektiv sind ..." (ebd., S. 28). Darüber hinaus betont Pöppel, dass die sinnliche Fundierung und die sinnlich-ästhetische Ordnung von Erfahrung hinsichtlich ihrer Sinnhaftigkeit und subjektiven Bedeutsamkeit strukturiert wird (vgl. ebd., S. 29 f.).

Folgt man Pöppels Überlegungen, kann für den Umgang mit Werken der Bildenden Kunst und alltagsästhetischen Phänomenen festgestellt werden, dass die Identitätsentwicklung auch im Rezeptionsprozess gefördert wird. Denn: will man Sinnzusammenhänge herstellen, sind Kenntnisse über die jeweiligen Inhalte hilfreich, Bedeutungskontexte werden recherchiert, subjektive Anmutung und emotionale Betroffenheit fließen ein, die Sinnessysteme und das Erinnerungswissen werden aktiviert. Vorstellungswissen hilft, die von Welsch genannten „Wirklichkeitsmodelle“ zu antizipieren. Insbesondere die eigene Produktionserfahrung Pöppel nennt diese implizites Handlungswissen - trägt dazu bei, die spezifischen Wirkungen eines ästhetischen Objekts zu entdecken und zu erschließen. Die mit dem Werk vermittelten Lebensentwürfe stellen eigene Identitätsvorstellungen in Frage, und helfen zugleich, unterschiedliche Wirklichkeitsvorstellungen in neue Zusammenhänge zu stellen: „Vor allem aber sind es die Bilder, die unsere Innenperspektive bestimmen ... Manchmal vermitteln uns Kunstwerke eine Ahnung davon, welche Bilder wir in uns tragen. Wir erkennen dann gleichsam ein fremdes Bild in uns wieder.“ (Pöppel 2000, S. 31). 
Die produktive und rezeptive Beschäftigung mit Beispielen aus der Kunst kann dazu beitragen, dass die Schülerinnen und Schüler sich auch mit ihren eigenen Lebensentwürfen und Identitätskonstruktionen auseinandersetzen. Die Werke bieten inhaltliche und gestalterische Anregungen, die Schülerinnen und Schüler in ihrer Identitätsfindung unterstützen, etwa wenn es gelingt eindrückliche und intersubjektiv verstehbare Menschen, Orte oder Situationen mit der Fotokamera einzufangen, die anders vielleicht nicht formuliert werden können. Identität und Ich-Stärke entstehen durch die Erfahrung der eigenen Selbstwirksamkeit, wenn ich etwas herstelle, das eine anspruchsvolle Lösung aufweist und somit subjektive Zufriedenheit auslöst. Thematisch kann die eigene Person im Zentrum des Interesses stehen, die sich im Vergleich mit anderen, im Spiegel der Gesellschaft, im Kontext anderer kultureller Hintergründe oder historischer Zusammenhänge konstituiert. Nur durch die Reflexion des Selbst in der Auseinandersetzung mit dem Anderen kann Identität entstehen. Das bedeutet, erst wenn es gelingt, die Schülerinnen und Schüler mit geeigneten Themenstellungen und Methoden zur Selbstreflexion anzuregen, werden identitätsfördernde Prozesse in Gang gesetzt.

Für Grundschulkinder kann bereits eine neue Materialerfahrung identitätsstiftend wirken, da durch den Umgang mit verschiedenen Materialien die Entwicklungsgrundlagen im triebdynamischen, emotionalen und sinnlichen Bereich, die zum Aufbau kognitiver Kompetenzen Voraussetzung sind, gefördert werden. Für Jugendliche sind es eher mediale Vorbilder und Idole, existenzielle Themen (Liebe, Tod, Hass, Freundschaft usw.) und gesellschaftliche Erwartungen, die in der Auseinandersetzung damit zur Identitätsfindung beitragen. In vielerlei Schulklassen kann die kulturelle Identität von besonderer Bedeutung sein, die es in den Blick zu nehmen gilt. Kinder und Jugendliche mit Migrationshintergrund beherrschen eine weitere Sprache, Rollenerwartungen zu Männer- und Frauenbildern oder verschiedenen Religionen können thematisiert werden. Allerdings geht es nicht darum, vermeintlich kulturell geprägte Klischees zu reproduzieren, sondern individuelle Möglichkeiten der Selbstverwirklichung - Bezug nehmend auf die eigenen Stärken, Wünsche und Ausdrucksbedürfnisse - in den Vordergrund zu rücken. Eine eigene „Klassenidentität“ auszubilden (Petzel 2010, S. 100 ff.), kann Motor sein für Gemeinschaftsaufgaben im Kunstunterricht oder Themenstellungen, die das untereinander Kennenlernen fördern. Jedes einzelne Kind muss sich innerhalb einer neuen Klasse zunächst positionieren; dann werden Kleingruppen gebildet, bis schließlich das Zusammenwachsen beginnt. Alle Formen des gemeinsamen Spiels (Schattentheater, Darstellendes Spiel, Maskenspiel usw.) sind geeignet, diesen Prozess zu unterstützen, denn ein gemeinsames Ziel verbindet.

In welchem Ausmaß der Kunstunterricht die Identitätsentwicklung fördern und stärken kann, bleibt letztlich offen: Der Zugewinn an Identität ist nicht messbar. Auch ist der Kunstunterricht kein Allheilmittel, um gesellschaftlich erzeugte Defizite zu kompensieren, doch er kann dazu beitragen, die Wege zum Selbst zu öffnen und Reflexionsprozesse einzuleiten, die das Ich im Anderen erkennen lassen.

Anmerkung: Die Abschnitte 3-5 dieses Aufsatzes gehen in einigen Teilen zurück auf: Kirchner, Constanze: Identität und Ausdruck. Gestalterisches Probehandeln, Selbstinszenierung und Rollenspiele. In: Kunst+Unterricht 366/367/2012, S. 4 - 21 


\section{Literatur}

Bal, Mieke (2013): Mise en scène: Zur Inszenierung von Subjektivität. In: Früchtl, Josef/ Zimmermann, Jörg (Hrsg.): Ästhetik der Inszenierung. (1. Aufl. 2001). Frankfurt am Main: Suhrkamp, S. 198-221.

Baldauf, Brigitte (2012): „Me, myself and I“. Inszenierte Fotografie als Suche nach einem eigenen Standort. In: Kunst+Unterricht 366/367 2012, S. 58-59.

Buholzer, Alois/ Kummer/Wyss, Annemarie (Hrsg.) (2010): Alle gleich - alle unterschiedlich! Zum Umgang mit Heterogenität in Schule und Unterricht. Seelze-Velber: Friedrich.

Drühl, Sven (o.J.): Androgynität in der Gegenwartskunst. In: Schreibkraft - das Feuilletonmagazin. Graz: Edition Schreibkraft, http://schreibkraft.adm.at/ausgaben/02-wiederkehr/androgynitat-in-dergegenwartskunst (Zugriff am 12.04.2012).

Erikson, Erik H. (1973): Identität und Lebenszyklus. Frankfurt am Main: Suhrkamp Wissenschaft.

Eßer, Anne (2012): Selbstinszenierung im Kunstunterricht. Ikonen der Weiblichkeit und Männlichkeit. In: Kunst+Unterricht 366/367 2012, S. 62-70.

Fend, Helmut (2005): Entwicklungspsychologie des Jugendalters. (Nachdruck der 3. Aufl. 2003). Wiesbaden: VS.

Fischer-Lichte, Erika (2004): Ästhetik des Performativen. Frankfurt am Main: Suhrkamp 2004.

Frey, Hans-Peter/ Haußer, Karl (Hrsg.) (1987): Identität. Entwicklungen psychologischer und soziologischer Forschung. Stuttgart: Thieme.

Früchtl, Josef/ Zimmermann, Jörg (Hrsg.) (2013): Ästhetik der Inszenierung. (1. Aufl. 2001). Frankfurt am Main: Suhrkamp.

Früchtl, Josef/ Zimmermann, Jörg (2013): Ästhetik der Inszenierung. Dimensionen eines gesellschaftlichen, individuellen und kulturellen Phänomens. In: Früchtl, Josef/ Zimmermann, Jörg (Hrsg.): Ästhetik der Inszenierung. (1. Aufl. 2001). Frankfurt am Main: Suhrkamp, S. 9-47

Goffman, Erving (2003): Wir alle spielen Theater. (Original: The presentation of self in everyday life, 1959). München: Piper.

Hafeneger, Benno (2003): Jugendkulturelle Modernisierung. Subjektbezug in Lernen und Bildung. Schwalbach/Ts.: Wochenschau.

Haußer, Karl (1995): Identitätspsychologie. Berlin, Heidelberg, New York: Springer.

Heinzlmaier, Bernhard (2010): Jugendkulturen in der Postmoderne - gesellschaftliche Veränderungen und ihre Auswirkungen auf das ästhetische Verhalten. In: Kirchner, Constanze/ Kirschenmann, Johannes/ Miller, Monika (Hrsg.): Kinderzeichnung und jugendkultureller Ausdruck. Forschungsstand Forschungsperspektiven. München: kopaed, S. 135-144.

Holschbach, Susanne (2011): Geschlechterrollen. In: Stooss, Toni/ Ruelfs, Esther (Hrsg.): Rollenbilder Rollenspiele. Katalog zur gleichnamigen Ausstellung im Museum der Moderne Salzburg Mönchsberg 23.07. - 30.10.2011. München: Hirmer

Jauß, Hans Robert (1994): Wege des Verstehens. München: Fink.

Keupp, Heiner (2009): Identitätskonstruktionen in der spätmodernen Gesellschaft - Riskante Chancen bei prekären Ressourcen. In: Theunert, Helga (Hrsg.): Jugend - Medien - Identität. Identitätsarbeit Jugendlicher mit und in Medien. München: kopaed.

Keupp, Heiner/ Ahbe, Thomas/ Gmür, Wolfgang/ Höfer, Renate/ Mitzscherlich, Beate/ Kraus, Wolfgang/ Straus, Florian (1999): Identitätskonstruktionen. Das Patchwork der Identitäten in der Spätmoderne. Reinbek bei Hamburg: rororo.

Kirchner, Constanze (2012): Identität und Ausdruck. Gestalterisches Probehandeln, Selbstinszenierung und Rollenspiele. In: Kunst+Unterricht 366/367 2012, S. 4-21.

Kirchner, Constanze/ Schiefer Ferrari, Markus/ Spinner, Kaspar H. (Hrsg.) (2006): Ästhetische Bildung und Identität. Fächerverbindende Vorschläge für die Sekundarstufe I und II. München: kopaed.

Kirschenmann, Johannes (2012): Identitätskonstruktionen im Musikvideo. Habitusofferten in Kunst und Medien. In: Kunst+Unterricht 366/367 2012, S. 71-77.

Krautz, Jochen (2012): Ich, Wir, Welt - Persönlichkeit und Beziehung in der Kunstdidaktik. In: Krautz, Jochen/ Schieren, Jost (Hrsg.): Persönlichkeit und Beziehung als Grundlage der Pädagogik. Weinheim/ Basel: Beltz/ Juventa.

$\mathrm{K}+\mathrm{U}=$ Kunst+Unterricht

Kunst+Unterricht 154, 1991: Papiertheater

Kunst+Unterricht 199, 1996: Maske und Maskenspiel

Kunst+Unterricht 274/275, 2003: Spiel

Kunst+Unterricht 280, 2004: Bio-Grafie

Kunst+Unterricht 317, 2008: Porträtieren 
Kunst+Unterricht 318, 2008: Porträtieren/ Material kompakt

Kunst+Unterricht 321/322, 2008: Theater

Kunst+Unterricht 329/330, 2009: Körper als Bild

Kunst+Unterricht 366/367, 2012: Identität und Ausdruck

Marcuse, Yvonne (2012): Motiv: Du selbst! Digitale Selbstinszenierung als Star. In: Kunst+Unterricht 366/367 2012, S. 41-43.

Neuenschwander, Markus P. (1996): Entwicklung und Identität im Jugendalter. Bern, Stuttgart, Wien: Paul Haupt.

Neumann-Braun, Klaus/ Richard, Birgit (2010): Szene-Hopping und kreativer Stilmix. Wechselnde Bündnisse für Spaß, Sinn und Selbstdarstellung. In: Schüler. Wissen für Lehrer: Szenen, Gruppen, Peers. SeelzeVelber: Friedrich.

Nummer-Winkler, Gertrud (2009): Identität und Wertbindung. In: Vierteljahreszeitschrift für wissenschaftliche Pädagogik, Heft 1/2009 (85. Jg.). Paderborn: Schöningh.

Petzel, Thomas (2010): Sich finden, sich binden, Klasse werden. Prozesse der Gruppenbildung. In: Schüler. Wissen für Lehrer: Szenen, Gruppen, Peers. Seelze-Velber: Friedrich.

Schäfer, Gerd E. (2003): Mit Bildern denken, in: Fröhlich, Volker/ Stenger, Ursula (Hrsg.): Das Unsichtbare sichtbar machen. Bildungsprozesse und Subjektgenese durch Bilder und Geschichten. Weinheim und München: Beltz/ Juventa.

Schuhmacher-Chilla, Doris (1995): Ästhetische Sozialisation und Erziehung. Zur Kritik an der Reduktion von Sinnlichkeit. Berlin: Reimer.

Schüler. Wissen für Lehrer 2010: Szenen, Gruppen, Peers. Seelze-Velber: Friedrich.

Schulze, Gerhard (1999): Kulissen des Glücks. Streifzüge durch die Eventkultur. Frankfurt am Main: Campus.

Shusterman, Richard (2013): Tatort: Kunst als Dramatisieren. In: Früchtl, Josef/ Zimmermann, Jörg (Hrsg.): Ästhetik der Inszenierung. (1. Aufl. 2001). Frankfurt am Main 2013, S. 126-143.

Seel, Martin (2013): Inszenieren als Erscheinenlassen. Thesen über die Reichweite eines Begriffs. In: Früchtl, Josef/ Zimmermann, Jörg (Hrsg.): Ästhetik der Inszenierung. (1. Aufl. 2001). Frankfurt am Main: Suhrkamp, S. 48-62.

Stooss, Toni/ Ruelfs, Esther (Hrsg.) (2011): Rollenbilder - Rollenspiele. Katalog zur gleichnamigen Ausstellung im Museum der Moderne Salzburg Mönchsberg 23.07. - 30.10.2011, München: Hirmer.

Theunert, Helga (Hrsg.) (2009): Jugend - Medien - Identität. Identitätsarbeit Jugendlicher mit und in Medien. München: kopaed.

Pöppel, Ernst (2000): Drei Welten des Wissens. Koordinaten einer Wissenschaft. In: Maar, Christa/ Obrist, Hans Ulrich/ Pöppel, Ernst (Hrsg.): Weltwissen - Wissenswelt. Das globale Netz von Text und Bild. Köln: DuMont, S. 21-39.

Rella, Christoph (2011): Facebook als neue Jugendreligion. In: Wiener Zeitung vom 20.06.2011. http://www.wienerzeitung.at/nachrichten/oesterreich/chronik/219352_Facebook-als-neueJugendreligion.html (Zugriff am 28.08.2013).

Welsch, Wolfgang (1993): Ästhetisches Denken. (3. Aufl.). Stuttgart: Reclam. 